\title{
Electronic transitions of tantalum monofluoride
}

Cite as: J. Chem. Phys. 146, 094308 (2017); https://doi.org/10.1063/1.4977215

Submitted: 07 December 2016 . Accepted: 11 February 2017 . Published Online: 03 March 2017

K. F. Ng, Wenli Zou (D), Wenjian Liu (D), and A. S.-C. Cheung

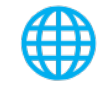

\section{ARTICLES YOU MAY BE INTERESTED IN}

Photodetachment spectroscopy of the beryllium oxide anion, $\mathrm{BeO}^{-}$

The Journal of Chemical Physics 146, 054301 (2017); https://doi.org/10.1063/1.4974843

The electric dipole moments in the ground states of gold oxide, AuO, and gold sulfide, AuS The Journal of Chemical Physics 146, 064307 (2017); https://doi.org/10.1063/1.4975816

Density matrix renormalization group (DMRG) method as a common tool for large activespace CASSCF/CASPT2 calculations

The Journal of Chemical Physics 146, 094102 (2017); https://doi.org/10.1063/1.4976644 


\title{
Electronic transitions of tantalum monofluoride
}

\author{
K. F. Ng, ${ }^{1}$ Wenli Zou, ${ }^{2}$ Wenjian Liu, ${ }^{3}$ and A. S.-C. Cheung ${ }^{1, \text { a) }}$ \\ ${ }^{1}$ Department of Chemistry, The University of Hong Kong, Pokfulam Road, Hong Kong, Hong Kong \\ ${ }^{2}$ Institute of Modern Physics, Northwest University, Xi'an, Shaanxi, China and Shaanxi Key Laboratory \\ for Theoretical Physics Frontiers, Xi'an, Shaanxi, China \\ ${ }^{3}$ College of Chemistry and Molecular Engineering, Peking University, Haidian, Beijing, China
}

(Received 7 December 2016; accepted 11 February 2017; published online 3 March 2017)

\begin{abstract}
The electronic transition spectrum of the tantalum monofluoride $(\mathrm{TaF})$ molecule in the spectral region between 448 and $560 \mathrm{~nm}$ has been studied using the technique of laser-ablation/reaction free jet expansion and laser induced fluorescence spectroscopy. The TaF molecule was produced by reacting laser-ablated tantalum atoms with sulfur hexafluoride gas seeded in argon. Twenty-two vibrational bands with resolved rotational structure have been recorded and analyzed, which were organized into seven electronic transitions. The $\mathrm{X}^{3} \Sigma^{-}\left(0^{+}\right)$state has been identified to be the ground state and the determined equilibrium bond length, $\mathrm{r}_{\mathrm{e}}$, and vibrational frequency, $\omega_{\mathrm{e}}$, are $1.8184 \AA$ and $700.1 \mathrm{~cm}^{-1}$, respectively. The low-lying $\Lambda$-S states and $\Omega$ sub-states of TaF were also theoretically studied at the MRCISD+Q level of theory with spin-orbit coupling. The $\Omega=0^{+}$and 2 sub-states from the ${ }^{3} \Sigma^{-}$and ${ }^{3} \Phi$ state have been found to be the ground and the first excited states, respectively, which agrees well with our experimental determinations. This work represents the first experimental investigation of the molecular structure of the TaF molecule. Published by AIP Publishing. [http://dx.doi.org/10.1063/1.4977215]
\end{abstract}

\section{INTRODUCTION}

The metal-fluorine (M-F) bond in transition metal fluoride (TMF) molecules has many characteristics. First, the chemical bonding between metal and fluorine is generally very strong ${ }^{1}$ and considered to be difficult to break in chemical reactions; however, it seems that this is not the case for some TMF. They participate in synthetic and catalytic reactions, leading to increasing interest in using TMF molecules in synthetic chemistry in recent years. ${ }^{2}$ Second, since the fluorine atom has the largest electro-negativity of all elements, the M-F bonds are usually highly polarized; however, the dipole moment of the TMF molecules eventually depends on the molecular structure and, very often, these molecules are non-polar. Third, the length of the M-F bond of the TMF molecules is subject to the valence state of the transition metal atom. As far as the Group V tantalum fluoride molecules are concerned, the $\mathrm{TaF}_{5}$ molecule has been used as a catalyst for ring opening synthesis; ${ }^{2}$ both $\mathrm{TaF}_{3}$ (planar) and $\mathrm{TaF}_{5}$ (trigonal bipyramids) are stable and non-polar molecules. From $\mathrm{x}$-ray crystal structural determination, the measured $\mathrm{Ta}-\mathrm{F}$ bond length was found to be $1.951 \AA$ for $\mathrm{TaF}_{3}$, and the bond length for equatorial and axial $\mathrm{F}$ atoms were determined to be 1.78 and $2.07 \AA$, respectively, for the $\mathrm{TaF}_{5}$ molecule. ${ }^{3,4}$ Recent densityfunctional theory (DFT) calculations by Ungerer et al. ${ }^{5}$ indicated that $\mathrm{TaF}_{5}$ is a closed-shell molecule and has no first order spin-orbit effects and the calculated equatorial Ta-F bond length is $1.89 \AA$, and the highest calculated harmonic frequency $\left(\mathrm{a}^{\prime}{ }_{1}\right)$ was $674 \mathrm{~cm}^{-1}$. It would be interesting to

\footnotetext{
a) Author to whom correspondence should be addressed. Electronic mail: hrsccsc@hku.hk. Tel.: (852) 2859 2155. Fax: (852) 28571586.
}

study the electronic structure of a single M-F bond to better understand the bonding characteristics of the $d$ orbitals. In addition, the five-fold degeneracy of the $d$ orbital leads to a wealth of low-lying electronic states with energies very sensitive to electron correlations in the molecule. We have investigated the bonding characteristics of the $\mathrm{Ta}-\mathrm{F}$ bond in this work.

Theoretical calculations of electronic structure of even diatomic molecules with transition metal elements are very challenging due to electronic correlation problems and a large number of open-shell electronic states arising from the incompletely filled $d$ orbitals of the transition metal atom. For more accurate results, high level calculations employing the state-ofthe-art methodology incorporating electron correlations and relativistic effects should be performed. Since tantalum is an element in the third transition period and with a large spinorbit interaction, the molecular states formed would also have large spin-orbit coupling (SOC) effects, which render the $\Lambda$-S state description of high spin multiplicity somewhat inadequate and also inappropriate. In this situation, the only good quantum number that could be used for labelling individual sub-states is the $\Omega$ value. It is necessary to perform $a b$ initio calculations on the spectroscopic properties of individual $\Omega$ spin components, which not only provides insights into the effect of the spin-orbit interactions and bonding characteristics of the molecule but the calculation results are also helpful for assigning the molecular transitions obtained from experiments.

The first theoretical investigation of the electronic structure of $\mathrm{TaF}$ was performed by Bauschlicher ${ }^{6}$ using density functional theory (DFT) and ab initio coupled cluster single and double $(\operatorname{CCSD}(\mathrm{T}))$ calculations. The ground state was predicted to be a ${ }^{3} \Sigma^{-}$state and the second lowest-lying state was 
$\mathrm{a}^{3} \Phi$ state, and their respective vibrational frequencies were found to be 704 and $675 \mathrm{~cm}^{-1}$. However, there has not been any experimental work on $\mathrm{TaF}$, but the experimental work on $\mathrm{TaCl}$ could be considered to be closely related to $\mathrm{TaF}^{7} \mathrm{In}$ this work, we report rotationally resolved electronic transition studies of $\mathrm{TaF}$ in the visible region using laser ablation/reaction supersonic free jet expansion and laser induced fluorescence (LIF) spectroscopy. Twenty-two vibrational bands were recorded and analyzed, which could be organized into seven electronic transition systems. Accurate molecular constants have been determined for the observed electronic states. In addition, high level theoretical calculations using the multireference configuration interaction have been performed, which provided spectroscopic properties on $\Lambda$-S states. Subsequently, the SOC was incorporated in the calculations of specific spectroscopic properties of individual $\Omega$ sub-states, and the results are compared with experimentally determined values.

\section{EXPERIMENTAL}

The laser ablation/reaction supersonic free jet expansion experimental setup and procedure for recording LIF spectra of gas-phase molecules has been described in our earlier publications, ${ }^{8,9}$ only a brief description of the experimental conditions will be given here. Laser pulses of $532 \mathrm{~nm}$ and $2 \mathrm{~mJ}$ from a Nd:YAG laser were focused on the surface of a tantalum metal rod to generate tantalum atoms. A pulsed valve, synchronized with an appropriate delay, released a gas mixture of $5 \% \mathrm{SF}_{6}$ in argon to react with $\mathrm{Ta}$ atoms and produce the TaF molecule. A tunable pulsed dye laser, operated with five Coumarin (C440, C460, C480, C503, and C540) dyes, was pumped by another Nd:YAG laser with the wavelength set to $355 \mathrm{~nm}$ producing laser output in the visible (448 $\mathrm{nm}$ to $560 \mathrm{~nm}$ ) region, which was used to excite the jet-cooled TaF molecule. The energy output of the tunable dye laser was about $1 \mathrm{~mJ}$ and the laser frequency was measured using a wavelength meter with accuracy $\pm 0.02 \mathrm{~cm}^{-1}$. The LIF signal was fed into a monochromator for recording the wavelength resolved fluorescence (WRF) spectrum, and the monochromator was also used as an optical filter to reduce the background scattering. The LIF signal was eventually detected by a photomultiplier tube and forwarded to a fast oscilloscope for averaging. The timing of all events was organized by a delay generator and the Nd:YAG laser and pulsed valve system were operated at $10 \mathrm{~Hz}$.

\section{AB INITIO CALCULATIONS}

The $\Lambda$-S states of TaF are calculated at the SA-CASSCF (state-averaged complete active space self-consistent field ${ }^{10,11}$ ) and the subsequent MRCISD+Q (internally contracted multireference configuration interaction with singles and doubles and Davidson's cluster correction ${ }^{12-14}$ ) levels of theory with the active space of 4 electrons in 6 orbitals, that is, the molecular orbitals corresponding to Ta $5 \mathrm{~d} 6 \mathrm{~s}$ are active. The lower orbitals from Ta $5 \mathrm{~s} 5 \mathrm{p}$ and $\mathrm{F} 2 \mathrm{~s} 2 \mathrm{p}$ are inactive but are also correlated, whereas the core orbitals with 2 and 60 core electrons for $\mathrm{F}$ and $\mathrm{Ta}$, respectively, are replaced by relativistic effective core potentials (RECPs). ${ }^{15,16}$ The standard aug-cc-pwCVQZ$\mathrm{PP}$ basis set ${ }^{16}$ is used for Ta, which has been optimized for the core-valence correlations in $5 \mathrm{~s} 5 \mathrm{p}$. For the fluorine atom, the aug-cc-pVQZ basis set ${ }^{17}$ is a little modified to combine with RECP: the first five steep primitive s-functions are excluded and then the rest of the primitive s- and p-functions are recontracted at the MRCISD level (see Ref. 18 for details). The SOC is calculated by the state-interaction approach ${ }^{19}$ at the SA-CASSCF level via the RECP spin-orbit operator, where the diagonal elements of the spin-orbit matrix are replaced by the above MRCISD+Q energies. The subgroup $C_{2 v}$ of $C_{\infty v}$ is applied.

The MOLPRO program package ${ }^{20}$ is used for all the above calculations, and the quantum numbers of the $\Omega$ states in $\mathrm{C}_{\infty \mathrm{v}}$ are recognized by a homemade utility program, whose concepts have been outlined in Table II of Ref. 21. The spectroscopic constants, including the adiabatic excitation energy $\left(T_{e}\right)$, equilibrium bond length $\left(R_{e}\right)$, vibrational constants $\left(\omega_{\mathrm{e}}\right.$ and $\left.\omega_{\mathrm{e}} \chi_{\mathrm{e}}\right)$, and rotational constant $\left(\mathrm{B}_{\mathrm{e}}\right.$ and $\left.\mathrm{B}_{0}\right)$, are obtained by solving the one-dimensional Born-Oppenheimer nuclear dynamics Schrödinger equation for ${ }^{181} \mathrm{Ta}^{19} \mathrm{~F}$ using the LEVEL program. ${ }^{22}$

\section{RESULTS AND DISCUSSION}

In the low resolution broadband scan, many molecular transition bands of the TaF molecule were observed, as shown in Figure 1. High resolution laser induced fluorescence spectra in the visible region between 435 and 560 $\mathrm{nm}$ were also recorded. Twenty-two vibrational bands were observed and analyzed rotationally. The bands analyzed are mostly $\Delta \Omega=0$ transitions with only one $\Delta \Omega=1$ transition, and the $\Omega$ values of the lower states are either 0 or 2 . These bands have been assigned to seven electronic transitions, namely, [18.6] $\Omega=0-\mathrm{X}^{3} \Sigma^{-}\left(0^{+}\right),[19.8] \Omega=0-\mathrm{X}^{3} \Sigma^{-}\left(0^{+}\right)$,

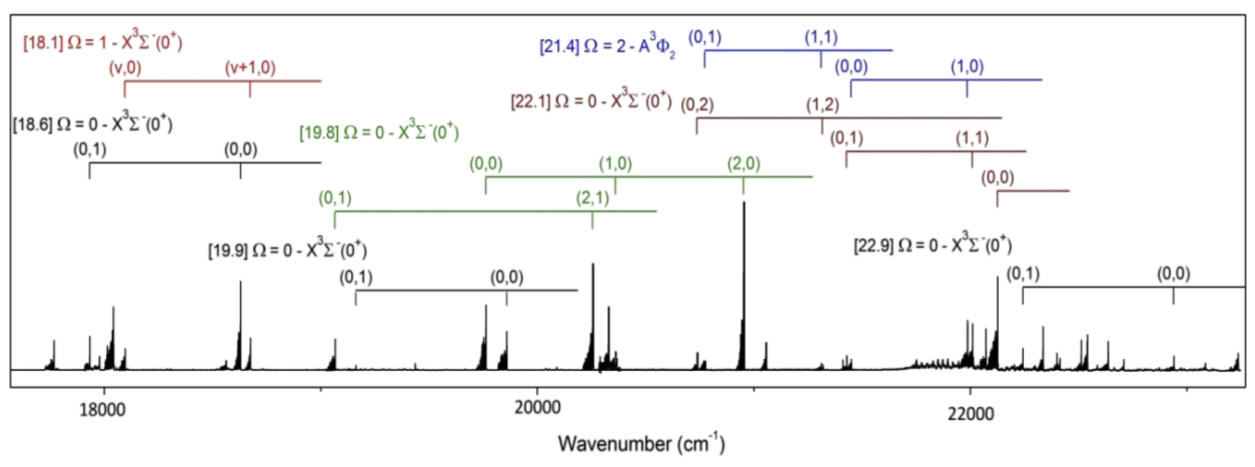

FIG. 1. Broadband scan of the TaF spectrum. 
$[19.9] \Omega=0-\mathrm{X}^{3} \Sigma^{-}\left(0^{+}\right),[22.1] \Omega=0-\mathrm{X}^{3} \Sigma^{-}\left(0^{+}\right),[22.9] \Omega$ $=0-\mathrm{X}^{3} \Sigma^{-}\left(0^{+}\right), \quad[18.1] \Omega=1-\mathrm{X}^{3} \Sigma^{-}\left(0^{+}\right)$, and $[21.4] \Omega=2$ $-\mathrm{A}^{3} \Phi_{2}$ systems. The observed and analyzed electronic transitions are summarized in Figure 2. As discussed earlier, since the SOC effect is large in the TaF molecule, it is appropriate to label the sub-state with the $\Omega$ value and the line positions were fit to a standard formula ${ }^{23}$

$$
\begin{aligned}
v= & v_{0}+B^{\prime} J^{\prime}\left(J^{\prime}+1\right)-D^{\prime}\left[J^{\prime}\left(J^{\prime}+1\right)\right] 2 \\
& -\left\{B^{\prime \prime} J^{\prime \prime}\left(J^{\prime \prime}+1\right)-D^{\prime \prime}\left[J^{\prime \prime}\left(J^{\prime \prime}+1\right)\right] 2\right\},
\end{aligned}
$$

where ' and " refers to the upper and lower states, respectively. The $v_{\mathrm{o}}$ is the band origin, and B and D are the rotational and centrifugal distortion constants. We performed least square fits of individual vibrational bands at the beginning and, eventually, in the final fit all bands were merged together to yield only one set of molecular constants for each vibrational level. Accurate molecular constants were determined for the nine electronic states involved and are listed in Table I. The observed transitions in this work are discussed individually. The line list of the measured line positions of the analyzed bands has been deposited to the journal archive as the supplementary material Appendix I.

\section{A. The $[19.8] \Omega=0-X^{3} \Sigma^{-}\left(0^{+}\right)$transition}

Five vibrational bands were recorded and rotationally analyzed. They are the $(0,0),(1,0),(2,0),(0,1)$, and $(2,1)$ bands of the [19.8] $\Omega=0-\mathrm{X}^{3} \Sigma^{-}\left(0^{+}\right)$transition. These bands show characteristics of a typical $\Omega^{\prime}=0-\Omega^{\prime \prime}=0$ transition, which contains only $\mathrm{P}$ and $\mathrm{R}$ branches and the corresponding first lines are the $\mathrm{P}(1)$ and $\mathrm{R}(0)$ lines. Figure 3 depicts the $(0,0)$ band of this transition. The lower state of this transition has been assigned to be the ground state, which is the $\Omega=0$ component of a ${ }^{3} \Sigma^{-}$state (i.e., $\mathrm{X}^{3} \Sigma^{-}\left(0^{+}\right)$), the rationale for this assignment is given in Section IV G. The equilibrium rotational constant, $\mathrm{B}_{\mathrm{e}}$, and the vibrational constant, $\omega_{\mathrm{e}}$, have been determined to be 0.2965 and $700.1 \mathrm{~cm}^{-1}$, respectively.

\section{B. The $[22.1] \Omega=0-\mathrm{X}^{3} \Sigma^{-}\left(0^{+}\right)$transition}

Five vibrational bands were recorded and rotationally analyzed. They are assigned to be the $(0,0),(0,1),(1,1),(0,2)$, and $(1,2)$ bands of the $[22.1] \Omega=0-X^{3} \Sigma^{-}\left(0^{+}\right)$transition system. Similar to the $[19.8] \Omega=0-X^{3} \Sigma^{-}\left(0^{+}\right)$transition, each band has $\mathrm{P}$ and $\mathrm{R}$ branches only, which is the characteristic

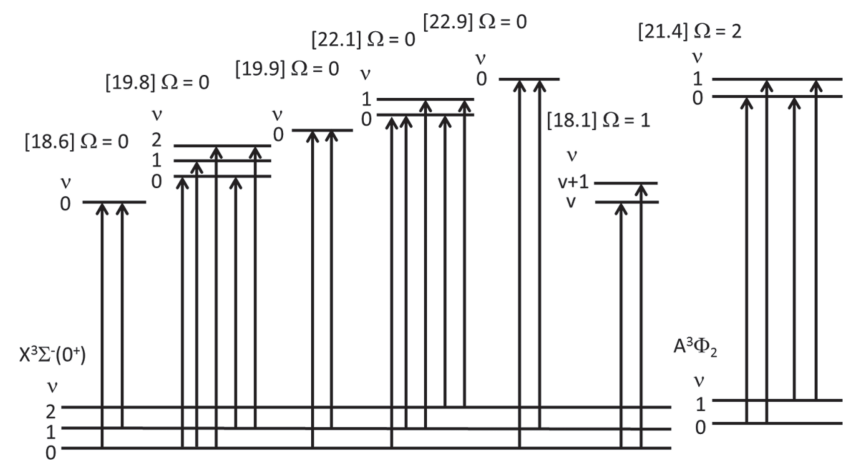

FIG. 2. Observed electronic transitions of TaF.
TABLE I. Molecular constants for the observed states of TaF $\left(\mathrm{cm}^{-1}\right)$.

\begin{tabular}{lcccc}
\hline \hline State & $\Omega$ & $v$ & $\mathrm{~T}_{v}$ & $\mathrm{~B}_{v}$ \\
\hline$[22.9]$ & 0 & 0 & $22937.47(1)$ & $0.2644(2)$ \\
{$[22.1]$} & 0 & 1 & $22704.47(1)$ & $0.2630(1)$ \\
& & 0 & $22123.51(5)$ & $0.2665(1)$ \\
{$[19.9]$} & 0 & 0 & $19856.69(4)$ & $0.2690(1)$ \\
{$[19.8]$} & 0 & 2 & $20950.05(5)$ & $0.2700(1)$ \\
& & 1 & $20357.69(4)$ & $0.2720(1)$ \\
{$[18.6]$} & 0 & 0 & $19759.82(5)$ & $0.2740(1)$ \\
{$[18.1]$} & 1 & $\mathrm{v}+1$ & $18626.33(2)$ & $0.2744(1)$ \\
& & 1 & $18672.36(1)$ & $0.2668(1)$ \\
$\mathrm{X}^{3} \Sigma^{-}$ & 0 & 2 & $1388.11(1)$ & $0.2685(1)$ \\
& & 1 & $697.02(1)$ & $0.2926(1)$ \\
& & 0 & 0 & $0.2963(1)$ \\
\hline State & $\Omega$ & $v$ & $\mathrm{~T}$ & $\mathrm{~B}_{v}$ \\
\hline$[21.4]$ & 2 & 1 & $\mathrm{a}+21982.90(6)$ & $0.2649(1)$ \\
& & 0 & $\mathrm{a}+21445.21(5)$ & $0.2685(1)$ \\
$\mathrm{A}^{3} \Phi_{2}$ & 2 & 1 & $\mathrm{a}+675.98(4)$ & $0.2821(1)$ \\
& & 0 & $\mathrm{a}$ & $0.2838(1)$ \\
\hline \hline
\end{tabular}

of a $\Omega^{\prime}=0-\Omega^{\prime \prime}=0$ transition. The measured line positions were fit to the formula in expression (1). Molecular constants obtained are reported in Table I; the lower state constants are the same as those from the $[19.8] \Omega=0-\mathrm{X}^{3} \Sigma^{-}\left(0^{+}\right)$transition which indicates that these two transitions share the same lower $\mathrm{X}^{3} \Sigma^{-}\left(0^{+}\right)$state.

\section{The $[18.1] \Omega=1-\mathrm{X}^{3} \Sigma^{-}\left(0^{+}\right)$transition}

Two vibrational bands at $18093 \mathrm{~cm}^{-1}$ and $18672 \mathrm{~cm}^{-1}$ were recorded and rotationally analyzed, which have been assigned as $(\mathrm{v}, 0)$ and $(\mathrm{v}+1,0)$ bands of the $[18.1] \Omega=1$ $-\mathrm{X}^{3} \Sigma^{-}\left(0^{+}\right)$transition. These bands show resolved $\mathrm{P}, \mathrm{Q}$, and $\mathrm{R}$ branches and the first lines of the branches are $\mathrm{P}(2), \mathrm{Q}(1)$, and $\mathrm{R}(1)$, respectively, which indicates that the transition is $\Omega^{\prime}=1-\Omega^{\prime \prime}=0$ transition. Molecular constants determined for the lower state are the same as the $\mathrm{v}=0$ level of the

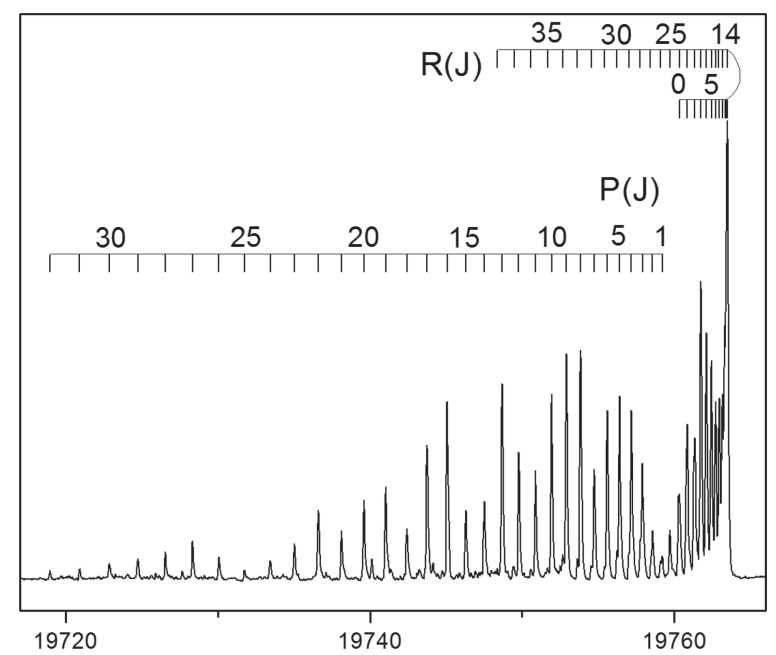

FIG. 3. The $(0,0)$ band of the $[19.8] \Omega=0-\mathrm{X}^{3} \Sigma^{-}\left(0^{+}\right)$transition of $\mathrm{TaF}$ showing only $\mathrm{P}$ and $\mathrm{R}$ branches. 


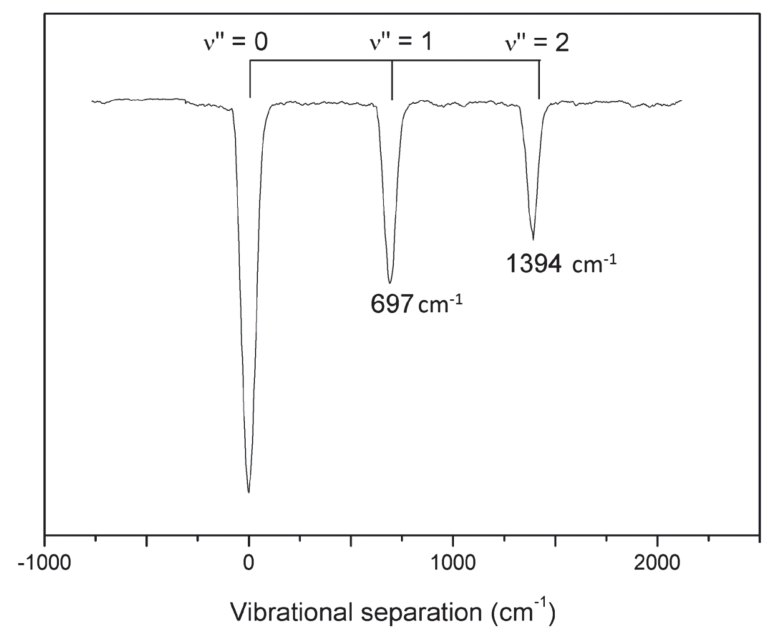

FIG. 4. Wavelength revolved fluorescence spectrum of the $(v+1,0)$ band of [18.1] $\Omega=1-\mathrm{X}^{3} \Sigma^{-}\left(0^{+}\right)$transition of TaF.

$\mathrm{X}^{3} \Sigma^{-}\left(0^{+}\right)$. Figure 4 is the wavelength resolved fluorescence spectrum of the $(v+1,0)$ transition, the lower state vibrational separation matches those of the $\mathrm{X}^{3} \Sigma^{-}\left(0^{+}\right)$state. However, for the upper state with the observation of only one vibrational level, we have not been able to assign the vibrational quantum number. Due to a tunable laser source limitation, despite the fact that bands were observed in lower resolution below $18000 \mathrm{~cm}^{-1}$, we could not record high resolution transitions lower in energy than the $18093 \mathrm{~cm}^{-1}$ band. This is the only $\Omega=1$ sub-state observed in this work. The vibrational separation of the upper state was determined to be $578.9 \mathrm{~cm}^{-1}$.

\section{The [21.4] $\Omega=2-\mathrm{A}^{3} \Phi_{2}$ transition}

Four vibrational bands, namely the $(0,0),(0,1),(1,0)$, and $(1,1)$ bands were observed and analyzed. Each band displays resolved $\mathrm{P}, \mathrm{Q}$, and $\mathrm{R}$ branches and the first lines of the branches are $\mathrm{P}(3), \mathrm{Q}(2)$, and $\mathrm{R}(2)$, respectively, which confirms that the band is a $\Omega^{\prime}=2-\Omega^{\prime \prime}=2$ transition. Figure 5 shows the $(0$, 1) band of the transition with all the three branches, and the intensity of the $\mathrm{Q}$ branch decreases very rapidly as J increases, which is consistent with a $\Delta \Omega=0$ transition. The lower state of this transition has been assigned to be the $\Omega=2$ component

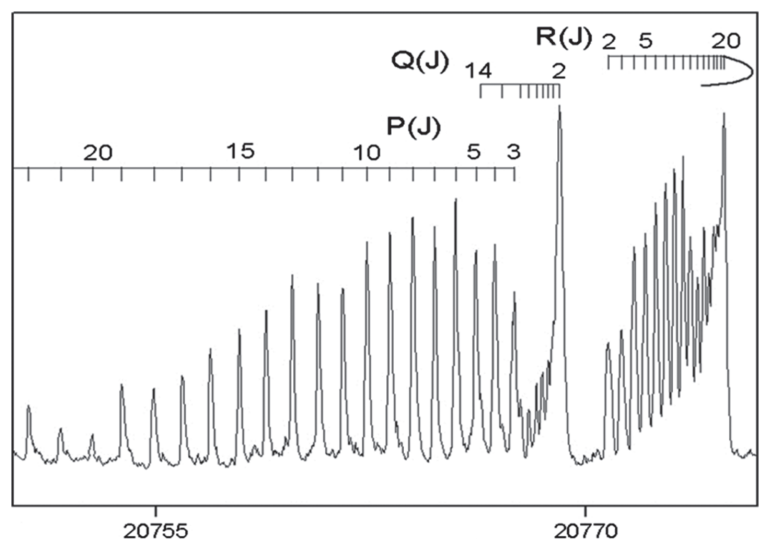

FIG. 5. The $(0,1)$ band of the $[21.4] \Omega=2-\mathrm{A}^{3} \Phi_{2}$ transition of TaF showing $\mathrm{P}, \mathrm{Q}$, and $\mathrm{R}$ branches. $\left({ }^{3} \Phi_{2}\right)$ of a low-lying ${ }^{3} \Phi$ state, the rationale for this assignment is given in Section IV G.

\section{E. The $v=0$ levels of the $[18.6] \Omega=0,[19.9] \Omega=0$, and $[22.9] \Omega=0$ states}

For the three transitions: the $[18.6] \Omega=0-\mathrm{X}^{3} \Sigma^{-}\left(0^{+}\right)$, $[19.9] \Omega=0-\mathrm{X}^{3} \Sigma^{-}\left(0^{+}\right)$, and [22.9] $\Omega=0-\mathrm{X}^{3} \Sigma^{-}\left(0^{+}\right)$transitions, only the $(0,0)$ and $(0,1)$ bands were observed. The spectrum consists of only the $\mathrm{P}$ and $\mathrm{R}$ branches indicating that they are $\Omega^{\prime}=0-\Omega^{\prime \prime}=0$ transitions. For the lower levels, the determined rotational constants and the vibrational separation are in good agreement with those observed for the $\mathrm{X}^{3} \Sigma^{-}\left(0^{+}\right)$ state. As far as the vibrational assignment of the upper state is concerned, we did search the lower energy region below the $(0,0)$ band and have not been able to observe any similar transition and, therefore, they are assigned as the $\mathrm{v}=0$ levels in each case.

\section{F. $A b$ initio results}

The potential energy curves (PECs) of low-lying $\Lambda$-S states of $\mathrm{TaF}$ are plotted in Figure 6, whereas in the supplementary material, theoretical and experimental levels of $\mathrm{Ta}^{+}$, PECs of low-lying $\Omega$ states, the potential energy data and the spectroscopic constants of $\Lambda$-S and $\Omega$ states, and the transition dipole moments of $\mathrm{TaF}$ are summarized. In order to test the accuracy of the calculations, at first the low-lying L-S and $\mathrm{J}$ terms of $\mathrm{Ta}^{+}$are calculated using the same method and are compared with the available experimental energies, ${ }^{24}$ as shown in Table S1 (Tables S1-S4 are in Appendix II, which are deposited as the supplementary material). It can be seen that the agreement is moderately good, which confirms the lower limit of accuracy of the molecular calculations because SOC will be partially quenched in the TaF molecule.

The low-lying $\Lambda$-S states of TaF are listed in Table S2 of the supplementary material. Different from the ground L-S level ${ }^{5} \mathrm{~F}$ of $\mathrm{Ta}^{+}$, the multiplicity of the ground $\Lambda-S$ state of $\mathrm{TaF}$ is triplet instead of quintet. Three $\Lambda$-S states $[1]^{1} \Sigma^{+},[1]^{1} \Gamma$, and $[1]^{3} \Sigma^{-}$associated with the $\mathrm{Ta}^{+} 5 \mathrm{~d}^{2}$ configurations have rather short equilibrium distances (around $1.8 \AA$ ), whereas the $r_{e}$ values of the $\mathrm{Ta}^{+} 5 \mathrm{~d}^{3}$ dominated states are about $1.9 \AA$ or much longer. On the contrary, in the late-d transition metal monohalides, it has been found that the $\mathrm{d}^{\mathrm{n}}$ dominated states have longer bond lengths and weaker bonding characters than the $\mathrm{d}^{\mathrm{n}+1}$ ones.

After perturbative treatment of SOC by the stateinteraction approach, PECs of the $\Omega$ states of TaF are plotted in Figure S1 in the supplementary material Appendix II, and the fitted spectroscopic constants and the dominant $\Lambda$-S states are collected in Table S3 where the value in parenthesis \{\} indicates the order of the calculated electronic sub-state with specific $\Omega$ value. Three $\Omega$ states with shorter bond lengths are obtained, i.e., $\{1\} 0^{+}$(mainly from $[1]^{3} \Sigma^{-}$), $\{1\} 1$ (from $[1]^{3} \Sigma^{-}$), and $\{2\} 4$ (from $[1]^{1} \Gamma$ ), but their bond lengths are slightly longer than their parent $\Lambda$-S states because of SOC interactions with other $\Lambda$-S states. The short bond state $[1]^{1} \Sigma^{+}$contributes heavily to several $\Omega=0^{+}$states but there is no $[1]^{1} \Sigma^{+}$-dominated $\Omega$ state. Among the $\Omega$ states, $\{1\} 0^{+}$is the ground one, and $\{1\} 2$ from 


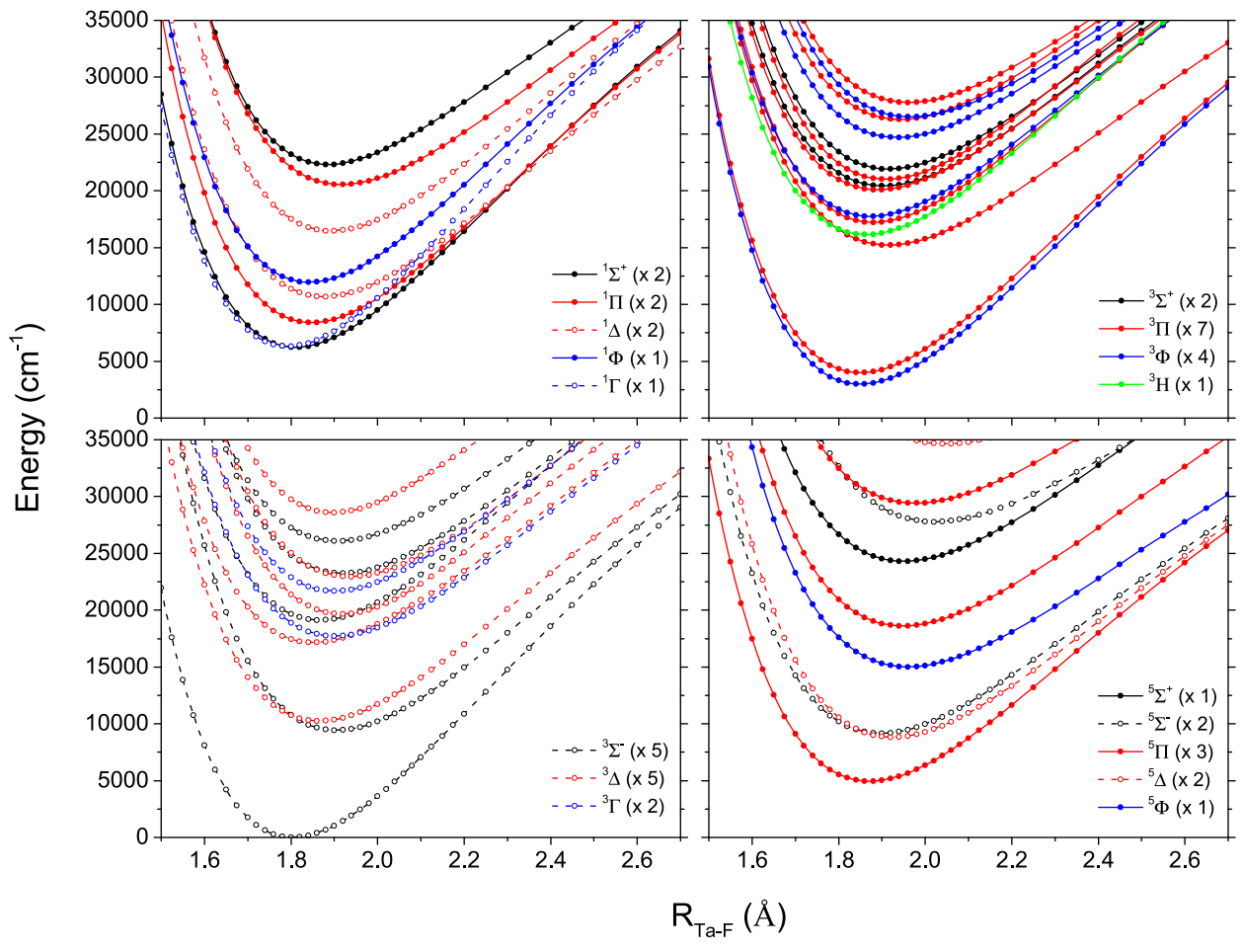

FIG. 6. Low-lying $\Lambda$-S states of TaF.
$[1]^{3} \Phi$ is the first excited one, being only about $100 \mathrm{~cm}^{-1}$ lower than the second excited state $\{1\} 1$. Table S4 lists the square of transition dipole moments $\left(\left|\mathrm{R}_{\mathrm{i}, \mathrm{j}}\right|^{2}\right)$ to some lower energy $\Omega$ states, which are calculated at the average bond length at $1.855 \AA$ of the lower $\Omega$ states. Large $\left|\mathrm{R}_{\mathrm{i}, \mathrm{j}}\right|^{2}$ values (usually $\geq 0.1$ a.u.) indicate strong spectral transitions. Some of the predicted transitions have been identified in this research, and our calculations suggest that many others should be detected in future experiments.

\section{G. Discussion}

The dominating electronic configurations for $\Lambda$-S molecular states obtained from ab initio calculations are the ${ }^{3} \Sigma^{-}$and ${ }^{3} \Phi$ and their molecular orbital occupations are as follows:

$$
\begin{gathered}
(1 \sigma)^{2}(1 \delta)^{2} \quad X^{3} \Sigma^{-}, \\
(1 \sigma)^{2}(1 \delta)^{1}(1 \pi)^{1} \quad \mathrm{~A}^{3} \Phi .
\end{gathered}
$$

As far as the calculated separation of these two states is concerned, the $\mathrm{A}^{3} \Phi$ state is higher in energy than the $\mathrm{X}^{3} \Sigma^{-}$state by $2985 \mathrm{~cm}^{-1}$. Including SOC effects, these two triplet states have spin-orbit components: the ${ }^{3} \Sigma^{-}$state has ${ }^{3} \Sigma^{-}\left(0^{+}\right)$and ${ }^{3} \Sigma^{-}(1)$ components and the ${ }^{3} \Phi$ state has ${ }^{3} \Phi_{2},{ }^{3} \Phi_{3}$, and ${ }^{3} \Phi_{4}$ components. Since the ${ }^{3} \Sigma^{-}$and ${ }^{3} \Phi$ are regular states, the sub-state with the smaller $\Omega$ value is lower in energy. When the calculations were included with the SOC effects, the spin-orbit components have energies as follows: the ${ }^{3} \Sigma^{-}\left(0^{+}\right)$sub-state is of the lowest energy (ground state), the ${ }^{3} \Sigma^{-}(1)$ is higher by $1786 \mathrm{~cm}^{-1}$, and the ${ }^{3} \Phi_{2},{ }^{3} \Phi_{3}$, and ${ }^{3} \Phi_{4}$ states are 1650 $\mathrm{cm}^{-1}, 4958 \mathrm{~cm}^{-1}$, and $6742 \mathrm{~cm}^{-1}$, respectively, above the ground state. As far as the experimental observation is concerned, we have no direct evidence showing that the $\Omega=0$ sub-state is lower in energy than the $\Omega=2$ sub-state; we notice that amongst the seven identified electronic transitions, six of them are from the same $\Omega=0$ sub-state, which is strongly indicative that this is the ground state. The calculation results are consistent and supportive of the experimental observation and we, accordingly, assign the ground state to be the ${ }^{3} \Sigma^{-}\left(0^{+}\right)$ and the next higher energy state is the $\mathrm{A}^{3} \Phi_{2}$ sub-state. We have been able to correlate the observed sub-states with those from theoretical calculations, which are summarized in Table II. The agreement between calculated and observed spectroscopic properties of these states is generally good. An exception is in the [21.4] $\Omega=2-\mathrm{A}^{3} \Phi_{2}$ or $\{16\} 2-\{1\} 2$ transition, where its $\left|R_{i, j}\right|^{2}$ is considerable but not very large (see Table $S 4$ of the supplementary material). This may be due to the orbital bias problem $^{25}$ in the state-averaged treatment with different Ta $5 \mathrm{~d}$ occupations, and therefore the transition dipole moments can be calculated only qualitatively. For comparison, although the lowest energy $\Omega=0$ and 2 sub-states of the $\mathrm{TaCl}$ molecule are quite similar to those of $\mathrm{TaF}^{6}$ the calculated energy for these two sub-states was reversed and the separation between them was too small to be conclusive. ${ }^{5}$ Which of these is the ground state of $\mathrm{TaCl}$ is still not clear.

It is interesting to note that the ground state equilibrium bond length for $\mathrm{TaF}$ determined from spectra is $1.8184 \AA$, which agrees quite well with the calculated values by Bauschlicher ${ }^{6}(1.815 \AA)$ and in this work $(1.814 \AA)$. The bond length of excited states of $\mathrm{TaF}$ is between 1.864 and $1.925 \AA$, which is larger than the ground state. Molecule with half- or fully-filled close shell configurations are usually associated with increased stability, reflected in shorter bond lengths. In the ground state $\mathrm{TaF}_{3}$ (open shell configuration), the Ta-F bond length is indeed longer than in diatomic TaF. As far as the $\mathrm{TaF}_{5}$ molecule is concerned, it has a trigonal bipyramidal structure and the Ta-F bond length on the equatorial plane is expected to be shorter than those on the axial positions. ${ }^{26}$ Since $\mathrm{TaF}_{5}$ has a close shell configuration, this argument would imply that it has a greater stability and should accordingly have shorter Ta-F bond lengths. The equatorial 
TABLE II. The observed and calculated spectroscopic properties of low-lying electronic states of $\mathrm{TaF}\left(\mathrm{cm}^{-1}\right)$.

\begin{tabular}{|c|c|c|c|c|c|c|}
\hline Electronic state & $\Omega$ & $\mathrm{T}_{0}$ & $r_{\mathrm{e}}(\AA)$ & $\omega_{\mathrm{e}}$ & $\mathrm{B}_{\mathrm{e}}$ & Components (\%) \\
\hline [22.9] & 0 & 22937.47 & $1.925^{\mathrm{a}}$ & & 0.2644 & \\
\hline$\{12\}$ & $0^{+}$ & 23608 & 1.901 & 646 & 0.2712 & $(4)^{3} \Pi(75)$ \\
\hline [22.1] & 0 & 22123.51 & $1.918^{\mathrm{a}}$ & $581.0^{\mathrm{b}}$ & 0.2665 & \\
\hline$\{11\}$ & $0^{+}$ & 22550 & 1.889 & 623 & 0.2748 & $(3)^{3} \Sigma^{-}(51)+(2)^{5} \Pi(13)$ \\
\hline [21.4] & 2 & $a+21982.90$ & $1.911^{\mathrm{a}}$ & $537.7^{\mathrm{b}}$ & 0.2685 & \\
\hline$\{16\}$ & 2 & 23170 & 1.912 & 666 & 0.2681 & $\begin{array}{c}(4)^{3} \Pi(33)+(2)^{5} \Pi(25)+(2)^{3} \Pi(11) \\
+(2)^{3} \Delta(11)\end{array}$ \\
\hline [19.9] & 0 & 19856.69 & $1.909^{\mathrm{a}}$ & & 0.2690 & \\
\hline$\{10\}$ & $0^{+}$ & 21582 & 1.924 & 587 & 0.2649 & $(5)^{3} \Pi(53)+(2)^{5} \Pi(11)+(4)^{3} \Sigma^{-}(10)$ \\
\hline$[19.8]$ & 0 & 19759.82 & 1.891 & 603.3 & 0.2750 & \\
\hline$\{9\}$ & $0^{+}$ & 20151 & 1.900 & 626 & 0.2715 & $(3)^{3} \Pi(39)+(2)^{5} \Pi(37)+(5)^{3} \Pi(16)$ \\
\hline [18.6] & 0 & 18626.33 & $1.890^{\mathrm{a}}$ & & 0.2744 & \\
\hline$\{8\}$ & $0^{+}$ & 19208 & 1.900 & 597 & 0.2714 & $\begin{array}{c}(3)^{3} \Pi(41)+(3)^{3} \Sigma^{-}(22)+(2)^{5} \Pi(16) \\
+(5)^{3} \Pi(10)\end{array}$ \\
\hline [18.1] & 1 & 18093.46 & 1.911 & $578.9^{\mathrm{c}}$ & 0.2685 & \\
\hline$\{12\}$ & 1 & 18516 & 1.908 & 594 & 0.2693 & $(3)^{3} \Pi(39)+(2)^{3} \Pi(33)$ \\
\hline $\mathrm{A}^{3} \Phi_{2}$ & 2 & $a$ & 1.864 & $676.0^{\mathrm{b}}$ & 0.2821 & \\
\hline$\{1\}$ & 2 & 1650 & 1.853 & 681 & 0.2855 & $(1)^{3} \Phi(91)$ \\
\hline $\mathrm{X}^{3} \Sigma^{-}$ & 0 & 0 & 1.818 & 700.1 & 0.2965 & \\
\hline$\{1\}$ & $0^{+}$ & 0 & 1.814 & 701 & 0.2980 & $(1)^{3} \Sigma^{-}(73)+(1)^{1} \Sigma^{+}(14)+(1)^{3} \Pi(12)$ \\
\hline
\end{tabular}

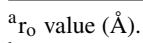

${ }^{\mathrm{b}} \Delta \mathrm{G}_{1 / 2}$ value.

${ }^{\mathrm{c}}$ Vibrational separation.

bond length of $\mathrm{TaF}_{5}$ is $1.78 \AA{ }^{4}{ }^{4}$ which is indeed shorter than that of the open shell TaF molecule.

\section{SUPPLEMENTARY MATERIAL}

See supplementary material for the line list of observed electronic transitions (Appendix I), theoretically calculated molecular parameters for electronic states (Appendix II) and associated data for the potential energy curves of low-lying electronic states (Appendix III).

\section{ACKNOWLEDGMENTS}

The work described here was supported by a grant from the University Research Committee of the University of Hong Kong.

${ }^{1}$ R. Murugavel and H. W. Roesky, Angew. Chem., Int. Ed. Engl. 36, 477 (1997).

${ }^{2}$ V. A. Petrov, Synthesis 2225 (2002).

${ }^{3}$ V. Gutmann and K. H. Jack, Acta Crystallogr. 4, 244 (1950).

${ }^{4}$ A. J. Edwards, J. Chem. Soc. 3714 (1964).

${ }^{5}$ M. J. Ungerer, C. G. C. van Sittert, D. J. van der Westhuizen, and H. M. Krieg, Comput. Theor. Chem. 1090, 112 (2016).

${ }^{6}$ C. W. Bauschlicher, Jr., J. Phys. Chem. A 104, 5843 (2000).

${ }^{7}$ R. S. Ram, W. S. Hopkins, A. G. Adam, and P. F. Bernath, J. Mol. Spectrosc. 232, 358 (2005).
${ }^{8}$ H. F. Pang and A. S.-C. Cheung, Chem. Phys. Lett. 471, 194 (2009).

${ }^{9}$ Q. Ran, W. S. Tam, C. Ma, and A. S.-C. Cheung, J. Mol. Spectrosc. 198, 175 (1999).

${ }^{10}$ H.-J. Werner and P. J. Knowles, J. Chem. Phys. 82, 5053 (1985).

${ }^{11}$ P. J. Knowles and H.-J. Werner, Chem. Phys. Lett. 115, 259 (1985).

${ }^{12}$ H.-J. Werner and P. J. Knowles, J. Chem. Phys. 89, 5803 (1988).

${ }^{13}$ P. J. Knowles and H.-J. Werner, Chem. Phys. Lett. 145, 514 (1988).

${ }^{14}$ P. J. Knowles and H.-J. Werner, Theor. Chim. Acta 84, 95 (1992).

${ }^{15}$ L. F. Pacios and P. A. Christiansen, J. Chem. Phys. 82, 2664 (1985).

${ }^{16}$ D. Figgen, K. A. Peterson, M. Dolg, and H. Stoll, J. Chem. Phys. 130, 164108 (2009).

${ }^{17}$ T. H. Dunning, Jr., J. Chem. Phys. 90, 1007 (1989).

${ }^{18}$ W. Zou and W. Liu, J. Comput. Chem. 30, 524 (2009).

${ }^{19}$ A. Berning, M. Schweizer, H.-J. Werner, P. J. Knowles, and P. Palmieri, Mol. Phys. 98, 1823 (2000).

${ }^{20}$ H.-J. Werner, P. J. Knowles, G. Knizia, F. R. Manby, M. Schütz et al., MOLPRo, version 2010.1, a package of ab initio programs, 2010, see http://www.molpro.net.

${ }^{21}$ W. Zou and W. Liu, J. Chem. Phys. 124, 154312 (2006).

${ }^{22}$ R. J. Le Roy, J. Quant. Spectrosc. Radiat. Transfer 186, 167 (2017).

${ }^{23} \mathrm{G}$. Herzberg, Spectra of Diatomic Molecules (Van Nostrand, New York, 1950).

${ }^{24}$ A. Kramida, Yu. Ralchenko, J. Reader, and, NIST Atomic Spectra Database (version 5.3) (National Institute of Standards and Technology, Gaithersburg, MD, 2015), URL: http://physics.nist.gov/asd.

${ }^{25}$ C. W. Bauschlicher, Jr., S. P. Walch, and S. R. Langhoff, in Quantum Chemistry: The Challenge of Transition Metals and Coordination Chemistry, NATO ASI Series C: Mathematical and Physical Sciences Vol. 176, edited by A. Veillard (D. Reidel Publishing Company, Dordrecht, 1986), pp. 15-35.

${ }^{26}$ R. L. DeKock and H. B. Gray, Chemical Structure and Bonding (University Science Book, 1989). 